\title{
Validation of a thermal bias control technique for Coda Wave Interferometry (CWI)
}

\author{
Yuxiang Zhang ${ }^{\mathrm{a}, *}$, Odile Abraham ${ }^{\mathrm{a}}$, Vincent Tournat ${ }^{\mathrm{b}}$, Alain Le Duff ${ }^{\mathrm{c}}$, Bertrand Lascoup ${ }^{\mathrm{d}}$, Ahmed Loukili ${ }^{\mathrm{e}}$, \\ Frédéric Grondin ${ }^{\mathrm{e}}$, Olivier Durand ${ }^{\mathrm{a}}$
}

${ }^{a}$ LUNAM Université, IFSTTAR, MACS, CS4, F-44344 Bouguenais Cedex, France

${ }^{\mathrm{b}}$ LAUM, CNRS 6613, Université du Maine, Avenue O. Messiaen, 72085 Le Mans, France

${ }^{\mathrm{C}}$ Groupe ESEO, 4 rue Merlet de la Boulaye, 49009 Angers, France

${ }^{\mathrm{d}}$ ESTACA, Parc Universitaire Laval-Changé, Rue Georges Charpak, 53061 Laval, France

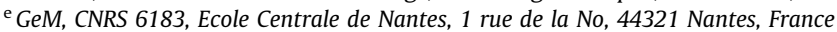

\begin{abstract}
The Coda Wave Interferometry (CWI) analysis serves to monitor the variation of propagation velocity in a heterogeneous medium with high precision $\left(10^{-3} \%\right.$ in relative terms). In combination with acoustoelastic theory, this type of analysis offers an NDT method for stress evaluation and/or damage detection. Since the CWI method is intended to evaluate extreme levels of accuracy, the presence of bias under certain circumstances can undermine evaluation results and/or test repeatability. In this paper, we offer a bias control technique involving the use of a second (reference) specimen for CWI analysis that is designed to compensate: (1) the thermally-induced velocity variation due to environmental temperature fluctuations; and (2) bias originating from experimental procedures. The presentation of this technique contains both a theoretical analysis and experimental protocol for the purpose of implementation. Furthermore, comparisons of experimental results have been included in order to demonstrate the utility of this bias control technique under laboratory conditions.
\end{abstract}

\section{Introduction}

The Coda Wave Interferometry (CWI) technique was originally developed by seismologists $[1,2]$ and later introduced into the NDT domain [3-5]. The use of multiple, scattered ultrasonic waves (i.e. acoustic coda waves) makes CWI analyses highly sensitive to the detection of a time-lapse perturbation in the propagation of elastic waves within materials. As a consolidated granular material, concrete exhibits strong heterogeneity; this characteristic serves to easily ensure the generation of acoustic coda waves, which then makes it feasible to conduct CWI analyses. The wave propagation velocity in concrete is correlated with its mechanical properties, thus yielding an indicator of the state of health of the concrete specimen [3]. In combining acoustoelastic theory, which links the structure's stress level to wave propagation velocity, CWI analysis is able to provide an accurate assessment of internal stress variations [6,7].

The high sensitivity of CWI analysis makes it possible to accurately study the mechanical response of concrete within a limited loading range, as well as to detect damage during the early stage. Since the CWI is intended for extremely accurate evaluations, any type of bias could undermine the result under certain circum-

\footnotetext{
* Corresponding author.

E-mail address: yuxiang.zhang@ifsttar.fr (Y. Zhang).
}

stances. A major bias of the CWI analysis stems from thermally-induced velocity changes. A temperature variation can cause changes in both geometry and elastic properties of the study material, thereby causing variations to the velocity evaluation result [8]. In studies on the mechanical properties of concrete, the thermally-induced velocity variation constitutes a bias that must not be neglected.

We are proposing herein an alternative bias control technique designed to lessen the impact of ambient temperature fluctuations on the CWI analysis result. This technique involves specific experimental protocols that introduce a reference specimen, combined with a differential analysis for the bias compensation; its performance has been evaluated by application to the study of concrete behavior under uniaxial tensile load within a laboratory setting. Experimental results demonstrate that this technique can efficiently reduce bias from both ambient temperature fluctuations and experimental procedures, thereby enhancing the precision of CWI analyses, allowing for the reproducibility of results and avoiding thermal interference.

Section 2 of this paper will provide the theoretical background; this material will include the general CWI analysis principle for evaluating constant velocity perturbations plus a detailed presentation of the Stretching method, i.e. a signal processing method used in CWI analysis. Acoustoelasticity theory will also be briefly discussed in this section. Section 3 will describe the bias control 
technique through a three-part format featuring: (1) our understanding of bias in the velocity evaluation, along with the theoretical model for compensation; (2) the measurement configuration of our experimental set-up, as an example of the implementation protocol; and (3) experimental results and several comparisons, to demonstrate the performance of this bias control technique.

\section{Theoretical background}

\subsection{CWI analysis for a constant velocity perturbation}

The Coda Wave Interferometry analysis has been designed to detect the time-scale perturbation of the propagation medium by using a multiple scattering medium as an interferometer [3]. A particular type of perturbation, i.e. a constant variation of propagation velocity, will be discussed in this study. The corresponding model was proposed for scalar wave propagation in 2002 [3] and then extended to elastic wave propagation [9]. This model is shown to nearly replicate physical perturbations under real conditions, such as temperature fluctuations $[10,3,11,12]$, external mechanical loading $[13,7,6,14]$ and changes in the state of health $[3,15,16]$ of the material. The use of CWI for monitoring these physical perturbations has proven feasible not only in the laboratory but also for in situ conditions $[8,5]$.

The estimation of velocity variation requires two coda signals $\left(h_{0}[t], h_{1}[t]\right)$ recorded, respectively before and after the velocity perturbation. By calculating the time lag $\Delta t$ at time $t_{0}$, the relative variation between propagation velocities when $\Delta v / v_{0}=-\Delta t / t_{0}$ can be obtained. Compared to traditional methods, which typically only use coherent waves, the introduction of acoustic coda waves (i.e. multiple scattered waves) increases sensitivity $[3,4,7,17]$, given that the time delay caused by constant velocity variation increases with the time of propagation. As this time lag widens, the estimated result becomes more accurate and reliable.

Fig. 1 presents $h_{0}[t]$ and $h_{1}[t]$ as two experimental signals recorded from concrete, respectively before and after a uniaxial tensile load has been applied. The difference in stress state causes a

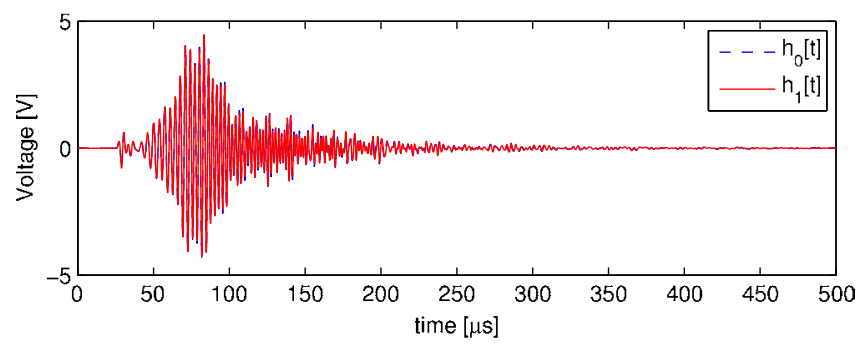

(a)

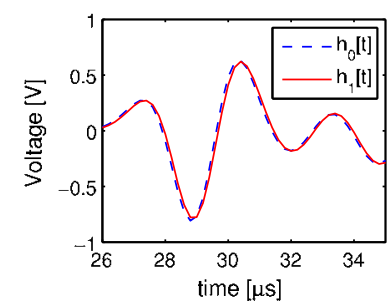

(b)

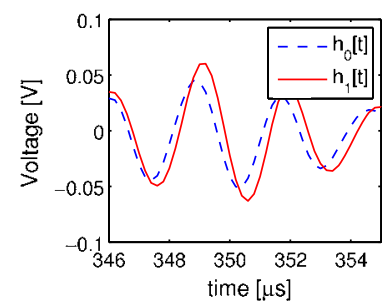

(c)
Fig. 1. Illustration of the increased sensitivity of coda waves to a velocity variation: (a) two coda signals $h_{0}[t]$ and $h_{1}[t]$ experimentally recorded before and after a velocity perturbation; the lower images present the same signals within a narrow time window ( $10 \mu$ s wide) centered at (b) an early propagation time of $30 \mu \mathrm{s}$; and (c) a later propagation time of $350 \mu \mathrm{s}$. variation in propagation velocity (see the discussion on acoustoelastic theory presented in the following paragraphs). As can be observed, two signals overlap during the early propagation times (Fig. 1b) when the time lag is hardly visible; after $300 \mu$ s of propagation however, the time lag due to this same velocity variation becomes larger and clearly visible (Fig. 1c).

In elastic wave propagation, acoustic energy is converted over different types of waves due to scattering effects. Multiple scattered waves therefore contain both shear $(\mathrm{S})$ and compressive $(\mathrm{P})$ waves. In a superposition of all multiple scattered waves arriving at the receiver, the coda signal contains contributions from both $S$ and $P$ waves. By counting the number of $P$ and $S$ modes, a model has been developed in 1982 to describe the acoustic energy partition between $S$ and $P$ waves on diffuse field $[18,19]$, and further used to interpret the CWI analysis result of velocity variation. According to [9], under the assumption of constant velocity variation and an equilibrium of acoustic energy, the CWI analysis result $\alpha=\Delta v / v$ can be considered as an effective velocity variation, which offers a weighted average value (see Eq. (1)) of the P-wave and Swave contributions. The weighting coefficients depend on the propagation velocities of both types of waves ( $v_{P}$ and $v_{\mathrm{S}}$ ).

$\frac{\Delta v}{v}=\frac{2 v_{S}^{3}}{2 v_{P}^{3}+v_{S}^{3}} \frac{\Delta v_{P}}{v_{P}}+\frac{2 v_{P}^{3}}{2 v_{P}^{3}+v_{S}^{3}} \frac{\Delta v_{S}}{v_{S}}$

For concrete fully saturated with water, $v_{S} \approx 2600 \mathrm{~m} / \mathrm{s}$ and $v_{P}-$ $\approx 4500 \mathrm{~m} / \mathrm{s}$ [20]. The CWI analysis result is primarily associated with the velocity variation of S-waves (Eq. (2)).

$\frac{\Delta v}{v}=0.09 \frac{\Delta v_{P}}{v_{P}}+0.91 \frac{\Delta v_{S}}{v_{S}}$

\subsection{Stretching}

Two signal processing approaches are available for the velocity variation estimation produced by the CWI analysis; these are known as Doublet [1] and Stretching [21]. According to a previous study [17] and under the assumption of a constant velocity variation, Stretching provides more stable and accurate results. For this reason, the Stretching approach has been chosen to carry out the CWI analysis as part of our study.

The Stretching approach starts by choosing a reference signal $h_{0}[t]$, which is to be recorded before the velocity perturbation. A decrease/increase in propagation velocity must then be simulated by means of stretching/compressing the time axis $t$ with a given dilatation rate $\tau_{i}$. By using the cross-correlation function within time-window $\left[t_{1}, t_{2}\right]$, the similarity between the stretched signal $h_{0}\left[t\left(1+\tau_{i}\right)\right]$ and the testing signal $h_{1}[t]$ (recorded after the velocity perturbation) can be quantified with a normalized coefficient $C C$ [22].

$C C_{\left(h_{0}, h_{1}\right)}^{\left(t_{1}, t_{2}\right)}\left(\tau_{i}\right)=\frac{\int_{t_{1}}^{t_{2}} h_{0}\left[t\left(1+\tau_{i}\right)\right] \cdot h_{1}[t] d t}{\sqrt{\int_{t_{1}}^{t_{2}} h_{0}^{2}\left[t\left(1+\tau_{i}\right)\right] d t \cdot \int_{t_{1}}^{t_{2}} h_{1}^{2}[t] d t}}$

The closer $C C\left(\tau_{i}\right)$ is to 1 , the greater the similarity in the two signals' waveforms. Following a grid search, $\alpha$ can be found among all values of $\tau_{i}$ that maximize the correlation coefficient $C C(\alpha)=\operatorname{Max}\left(C C\left(\tau_{i}\right)\right)$. Given that we have $\alpha=\left(v_{1}-v_{0}\right) / v_{0}=\Delta v / v_{0}$, the relative velocity variation can be derived. As an indicator of the decorrelation level between waveforms, the value of $C C(\alpha)$ depends on the record's signal-to-noise ratio, therefore consequently depends on the time-window $\left[t_{1}, t_{2}\right]$.

The choice of time-window $\left[t_{1}, t_{2}\right]$ is a key parameter in the CWI analysis. The start time of the window $t_{1}$ needs to be late enough to ensure the waves have been propagating for a long enough time to be sufficiently scattered and incoherent. The end of the time-win- 
dow $t_{2}$ is limited by the signal quality (i.e. the signal-to-noise ratio) since the acoustic energy becomes increasingly dissipated for longer propagation times. In our study, $t_{1}$ equals $300 \mu \mathrm{s}$, where time $t=0$ indicates the beginning of the excitation signal (Fig. 1a). The value of $t_{1}$ chosen to be more important than the minimum duration for ultrasonic waves in order to propagate a distance $L$ longer than 4 times the transport-mean free path $l^{*}\left(L>4 l^{*}\right)[23,24]$. The time-window is then selected from between $300 \mu$ s and $500 \mu \mathrm{s}$, in which a signal-to-noise ratio exceeds $40 \mathrm{~dB}$.

\subsection{Acoustoelastic theory}

The acoustoelastic effect refers to the phenomenon of wave propagation velocity variation due to the exposure to elastic strain. With respect to the linear approximation, Hooke's law describes the strain-stress relationship as $\varepsilon \cdot E=\sigma$, where $\sigma$ denotes the stress, $\varepsilon$ the strain and $E$ Young's elastic modulus. $E$ is constant according to this approximation, meaning it does not contribute to any velocity variation caused by a quasi-static stress. To understand the acoustoelastic effect and describe the stress-velocity relation, the material's nonlinear elasticity must be taken account. Among the different developed models [25], Murnaghan's classical nonlinear elastic theory [26] is shown applicable and widely used when stress level is relatively low.

Following the study of nonlinear elasticity by Murnaghan, the expression of acoustoelastic theory was extended by Hughes and Kelly [27] to describe the variation in propagation velocity due to applied elastic stress. This stress-velocity relationship has been further generalized in $[28,29]$ as:

$v_{i j}^{\sigma}=v_{i j}^{0}\left(1+\beta_{i j} \cdot \Delta \sigma\right)$

$v_{i j}^{\sigma}$ is the propagation velocity under stress, where $i$ and $j$ stand respectively for the propagation direction and polarization direction. $\beta_{i j}$ is the acoustoelastic coefficient, a nonlinear parameter that depends on both second-order elastic constants (Lamé's coefficients $\lambda$ and $\mu$ ) and third-order constants (Murnaghan's coefficients $m, n$ and $l)[26,28,29] . v_{i j}^{0}$ is the propagation velocity associated with the initial stress state; in the case of isotropic stress, it assumes the following form (where $\rho_{0}$ is the material density):

$v_{i \neq j}^{0}=\sqrt{\frac{\mu}{\rho_{0}}}$
$v_{i=j}^{0}=\sqrt{\frac{\lambda+2 \mu}{\rho_{0}}}$

Several studies on acoustoelastic effects have been carried out on concrete with a uniaxial compressive load; moreover, experimental results show a relatively strong agreement with acoustoelastic theory $[13,29,6,14,7]$. The linear relationship between the changes in applied stress and the velocity variation of coda waves has been confirmed in [7,30], while the concrete specimen remains in its elastic regime. As the dominant phenomenon, acoustoelastic theory will be introduced hereafter to understand the behavior of concrete under a low level tensile load ( $<1 \mathrm{MPa})$.

The CWI analysis with a Stretching approach can obviously yield a variation in effective propagation velocity through acoustoelasticity $\alpha$ (see Eq. (2)). However, due to the nature of coda waves, CWI analysis is incapable of identifying the velocity variation of each specific type of wave as $\alpha_{i j}=\Delta v_{i j} / v_{i j}^{0}$. Consequently, acoustoelastic coefficient cannot be acquired individually for each type of wave (as $\beta_{i j}$ in Eq. (2)). Instead, an effective coefficient $\beta$ can be obtained (Eq. (6)) as the weighted average value of all acoustoelastic coefficients $\beta=\sum e_{i j} \cdot \beta_{i j}$, where weight coefficients $e_{i j}$ depend on the energy distribution ratio of each type of wave.
$\alpha=\frac{\Delta v}{v_{0}}=\frac{v^{\sigma}-v^{0}}{v^{0}}=\beta \cdot \Delta \sigma$

\section{Bias control for CWI analysis}

The phenomenon of thermally-induced velocity variation has been observed on concrete [8,31] as well as on homogeneous materials [10,12], which makes environmental temperature fluctuation a physical bias in the propagation velocity analysis targeting the stress-induced velocity variation. In addition, the CWI analysis is beneficial because of its high sensitivity and capability of evaluating small velocity variations [7]. The experimental bias in this kind of estimation can undermine the precision and reliability required in order to analyze the CWI result. Along these lines, we are proposing herein a new alternative bias control protocol for CWI analyses. In a previous study [32], temperature effects have been discussed as a disturbance in acousto-ultrasonic monitoring techniques, and a number of proposals have been forwarded in a general way to minimize these effects. By studying these ideas along with the distinctive feature of CWI analyses, we are in a position to propose a highly feasible alternative bias control technique for CWI analyses.

\subsection{Theoretical model for bias compensation}

Let's assume that all variations in propagation velocity stem from three contributions: (1) the physical phenomenon under study, which in our case is associated with the impacts of applied stresses; (2) the thermal bias due to environmental temperature fluctuations; and (3) the experimental bias, which refers to all additional fluctuations of the CWI result originating from the entire measurement and signal processing procedure.

As presented in the section describing the CWI technique, velocity variation is evaluated from a time shift. Due to a perturbation in propagation velocity of the material, the wave arrival time shifts from $t_{0}$ to $t_{p}$. Under the assumption that propagation distance $l_{0}$ remains unchanged, we can easily derive the perturbed velocity $v_{p}$ as $\frac{v_{p}}{v_{0}}=\frac{t_{0}}{t_{p}}$, where $v_{0}$ is the unperturbed initial propagation velocity.

In reality, the applied stress on a structure definitely causes changes to the geometric dimensions, and this variation rate can be evaluated by its linear deformation $\varepsilon_{F}$. By assuming $\varepsilon_{F}$ to be uniform over the entire propagation medium, the propagation distance will change from $l_{0}$ to $l_{F}=l_{0}\left(1+\varepsilon_{F}\right)$. According to acoustoelastic theory, the wave propagation velocity also varies from $v_{0}$ to $v_{F}=v_{0}\left(1+\alpha_{F}\right)$. When combining changes in both distance and propagation velocity, the final arrival time $t_{F}$ is given as:

$t_{F}=\frac{l_{F}}{v_{F}}=\frac{l_{0}\left(1+\varepsilon_{F}\right)}{v_{0}\left(1+\alpha_{F}\right)}=t_{0} \frac{1+\varepsilon_{F}}{1+\alpha_{F}}$

Considering that both $\varepsilon_{F}$ and $\alpha_{F}$ are much smaller than 1 (in this study $\alpha_{F}<0.01$ and $\varepsilon_{F}$ is one order of magnitude smaller than $\alpha_{F}$, the measurement of propagation velocity under stress $\hat{v}_{F}$ takes the following form.

$\hat{v}_{F}=v_{0}\left(\frac{1+\alpha_{F}}{1+\varepsilon_{F}}\right) \simeq v_{0}\left(1+\alpha_{F}-\varepsilon_{F}\right)$

A temperature fluctuation exerts a similar influence on a propagation medium; as is the case in the presence of stress, it modifies the geometric dimensions and propagation velocity of the medium, eventually causing the propagation velocity measurement to shift from $v_{0}$ to $\hat{v}_{T}$, where $\varepsilon_{T}$ is the strain associated with temperature variation. A second-order term is also neglected, while a typical $\varepsilon_{F}$ for concrete lies on the order of $10^{-5}$, and in our study $\alpha_{T}$ is less than $10^{-3}$. 
$\hat{v}_{T}=v_{0}\left(\frac{1+\alpha_{T}}{1+\varepsilon_{T}}\right) \simeq v_{0}\left(1+\alpha_{T}-\varepsilon_{T}\right)$

In combining the influences from both applied stresses and temperature fluctuations, the propagation velocity measurement with temperature bias can be expressed with the second-order and third-order terms being neglected.

$$
\begin{aligned}
\hat{v}_{(F+T)} & =v_{0} \frac{\left(1+\alpha_{T}\right)\left(1+\alpha_{F}\right)}{\left(1+\varepsilon_{T}\right)\left(1+\varepsilon_{F}\right)} \\
& \simeq v_{0}\left(1+\alpha_{F}-\varepsilon_{F}+\alpha_{T}-\varepsilon_{T}\right)
\end{aligned}
$$

Let's consider two identical specimens experiencing the same thermal and experimental biases, with only one of them being subjected to an external stress. The specimen receiving all three velocity variation contributions is referred to as the test sample, while the other specimen exposed only to bias is the reference sample. By including the additional experimental bias term $\alpha_{N}$, we can express the CWI analysis results for both the test sample $\alpha_{t e s t}$ and reference sample $\alpha_{\text {ref }}$ as follows:

$$
\begin{aligned}
\alpha_{\text {test }} & =\frac{\hat{v}_{(F+T)}-v_{0}}{v_{0}}+\alpha_{N} \\
& =\alpha_{F}-\varepsilon_{F}+\alpha_{T}-\varepsilon_{T}+\alpha_{N} \\
\alpha_{\text {ref }} & =\frac{\hat{v}_{T}-v_{0}}{v_{0}}+\alpha_{N} \\
& =\alpha_{T}-\varepsilon_{T}+\alpha_{N}
\end{aligned}
$$

In considering that $\varepsilon_{F}$ is one order of magnitude smaller than the velocity variation $\alpha_{F}$, which is solely due to the impact of an applied stress on the propagation medium, can then be expressed as follows:

$\alpha_{F}(t)=\alpha_{\text {test }}(t)-\alpha_{\text {ref }}(t)$

\subsection{Implementation protocol}

In the experimental campaign, we performed this bias control technique by using two concrete specimens with the same geometry, same age, same initial moisture level and same mix composition (Table 1). Both specimens were cylindrical with a $0.11 \mathrm{~m}$ diameter and $0.45 \mathrm{~m}$ high. At the time of our experiment, the specimens were cast for over 3 months and their mechanical properties were stable. Both specimens are kept in a humid room at $100 \%$ relative humidity after casting to ensure their full saturation in water.

The entire experimental system was placed in a laboratory under controlled temperature $\left(20 \pm 2{ }^{\circ} \mathrm{C}\right)$ and relative humidity level $(50 \pm 5 \%)$. Nevertheless, in order to eliminate the influence of air humidity fluctuations on the propagation velocity, the two specimens were wrapped with a double layer of self-adhesive alumi-
Table 1

Concrete mix composition.

\begin{tabular}{ll}
\hline Constituents & Dosage $\left(\mathrm{kg} / \mathrm{m}^{3}\right)$ \\
\hline Gravel: G5/12.5 mm & 936.0 \\
Sand: 0/5 mm & 780.0 \\
Cement: CEMII 42.5 & 350.0 \\
Water & 219.5 \\
Superplasticizer & 1.9 \\
\hline
\end{tabular}

num paper to maintain their moisture level constant. As shown on the photographs in Fig. 2b, the test sample was placed in the mechanical press to be exposed to an applied stress, while the reference sample remained stress-free throughout the experiment (Fig. 2c). The specimens were placed adjacent to one another, as shown in Fig. 2a, so they could experience the same temperature fluctuations. Air temperature was measured with a Rotronic ${ }^{\circledR}$ thermometer placed between the two specimens.

For the ultrasonic acquisition system, two ultrasonic transducers were glued on opposite sides at mid-height of each specimen to serve as source and receiver. All transducers used in this experiment were of the same model, which covers a relatively small active zone (compared to wavelength at the central frequency), in order to reduce the risk of phase cancellation phenomenon [33]. The excitation signal for the source was a $200-\mu$ s chirp with frequency varying linearly from $200 \mathrm{kHz}$ to $800 \mathrm{kHz}$. In this frequency range, ultrasound wavelengths are comparable to the aggregate size, thus ensuring a strong multiple scattering regime.

To generate the identical experimental bias in records obtained from both the test and reference samples, the excitation signal was amplified to $200 \mathrm{~V}$ (peak-to-peak) then split and sent into both concrete specimens via ultrasonic transducers. The signals captured by ultrasonic receivers were then amplified (individually using two pre-amplifiers of the same model with the gains set to $20 \mathrm{~dB}$ ) and recorded with the same acquisition system at a sampling frequency of $5 \mathrm{MHz}$. The synchronization between excitation and acquisition was performed by a $10-\mathrm{MHz}$ reference clock signal (Fig. 3). To improve the signal-to-noise ratio, each record was averaged over 16 consecutive acquisitions with a repeat frequency of $30 \mathrm{~Hz}$. Since each record spanned such a short time (i.e. less than $1 \mathrm{~s}$ ), the propagation velocity in each record processed was considered constant. The CWI analysis results were extracted from these records by following the exact same signal processing procedures while keeping the same parameters.

\subsection{Experimental results}

The experimental performance of this bias control method is shown in Fig. 4. During this 90-min measurement, one loading-

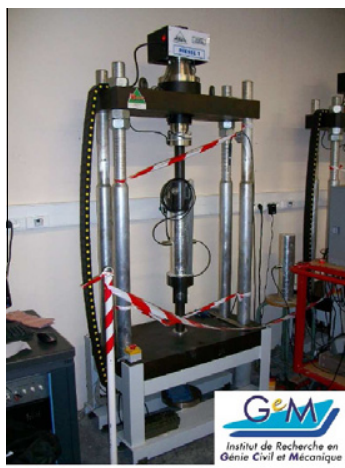

(a)

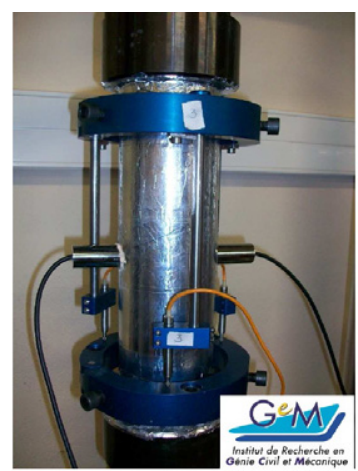

(b)

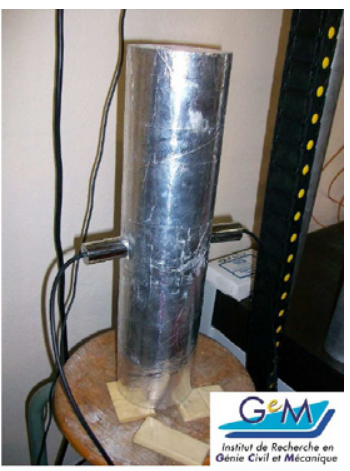

(c)

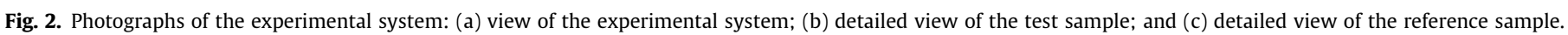




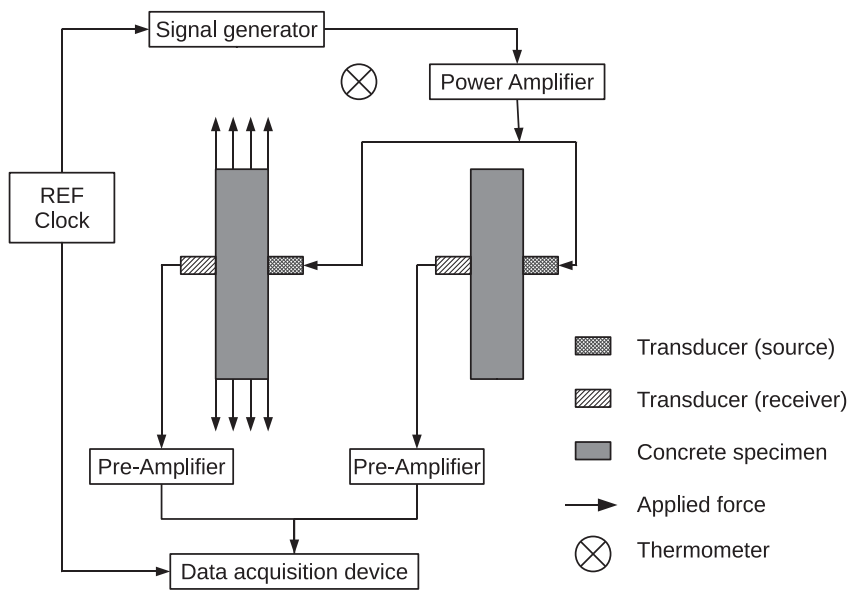

Fig. 3. Ultrasonic measurement system.

unloading test cycle was performed on the test sample. The magnitude of applied tensile force and air temperature fluctuations were plotted vs. time in Fig. 4a. The velocity variations in both specimens $\alpha_{\text {test }}$ and $\alpha_{\text {ref }}$ (i.e. without controlled bias) were plotted in Fig. $4 \mathrm{~b}$, in which $\alpha_{\text {ref }}$ indicates a periodic fluctuation with an ampli- tude of approx. $0.01 \%$. By referring to air temperature variation on the lower image (Fig. 4a), this fluctuation can be identified as a thermally-induced velocity variation. On the other hand, since the test sample has been subjected to a tensile load between 0 and $8 \mathrm{kN}$ (corresponding applied tension of $0.84 \mathrm{MPa}$ ), $\alpha_{\text {test }}$ follows the variation in loading magnitude and reveals a maximum decrease of $-0.12 \%$. During this test, with selected CWI analysis time-window, minimum $C C(\alpha)$ of 0.928 appears on the test sample under the maximum tensile load. This value indicates that signals are only slightly distorted, and all CWI analysis results of $\alpha$ are reliable. Because of this higher velocity variation (around $0.1 \%$ ) due to the stress effect, the influence of temperature fluctuation on $\alpha_{\text {test }}$ can be clearly observed only after the loading test (time $>45 \mathrm{~min}$ ). $\alpha_{\text {test }}$ after the loading test shows the same periodic fluctuation as $\alpha_{\text {ref, }}$ which is consistent with our assumption that the two specimens share the same thermal bias.

The CWI results with controlled bias $\alpha_{F}$ is illustrated in Fig. 5a, which theoretically indicates the velocity variation due only to the tensile force loading. The periodic velocity fluctuation due to thermal interference after the loading test has been eliminated, which means that the thermally-induced velocity variation has been compensated. In Fig. 5b, three curves vs. time show respectively $\alpha_{\text {ref, }}, \alpha_{\text {test }}$ and $\alpha_{F}$ on a magnified time scale (time $<8 \mathrm{~min}$ ). Both $\alpha_{\text {ref }}$ and $\alpha_{\text {test }}$ display a high-frequency, random-like fluctuation, which according to our theory reflects the experimental bias

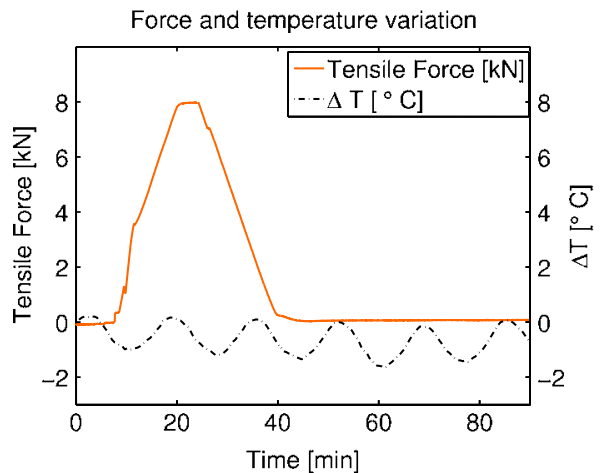

(a)

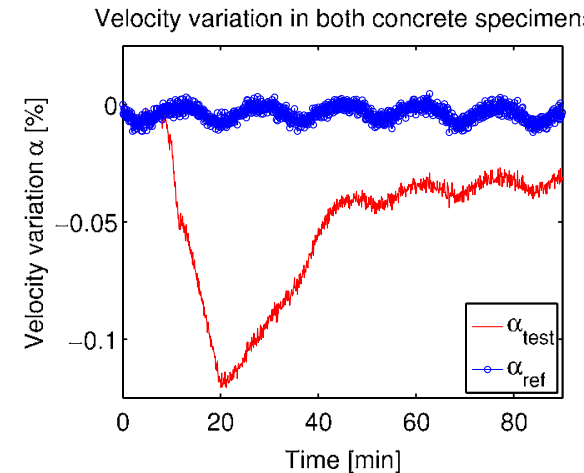

(b)

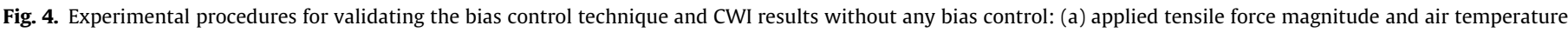

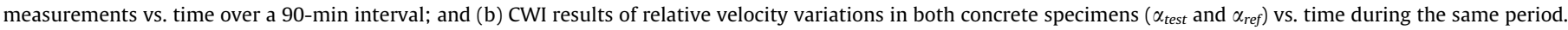

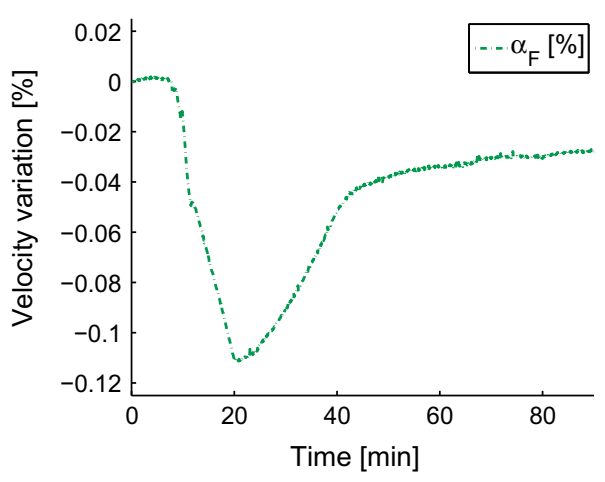

(a)

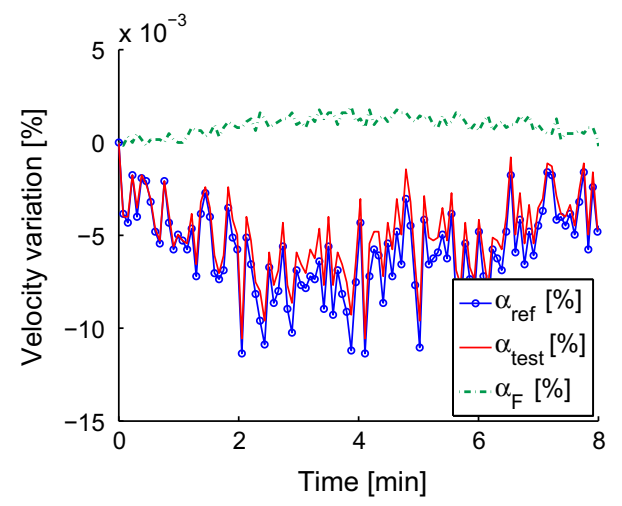

(b)

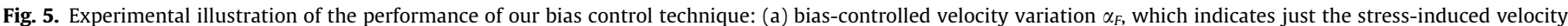

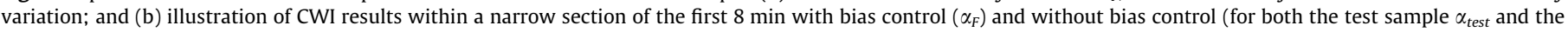
reference sample $\alpha_{\text {ref }}$ ); during this time window, all concrete specimens are stress-free. 
Table 2

Average values with standard error of velocity variation during the first $8 \mathrm{~min}$ of measurement for CWI results both with and without bias control as $\alpha_{F}$ and $\alpha_{\text {test. }}$.

\begin{tabular}{ll}
\hline$(\%)$ & Average velocity variation $\bar{\alpha}$ \\
\hline Without bias control & $0.00511 \pm 0.00217$ \\
With bias control & $0.00095 \pm 0.00022$ \\
\hline
\end{tabular}

due to measurement and signal processing procedures. With our bias control technique (see the section on Implementation proto$\mathrm{col}$ ), we would expect an identical experimental bias for the results of both specimens. This expectation is consistent with the observation that $\alpha_{\text {ref }}$ and $\alpha_{\text {test }}$ during this period are highly correlated, which yields a less noisy $\alpha_{F}$ curve. Since no force has been applied over this period (0-8 min), all velocity variations stem from thermal fluctuations and experimental bias, which according to our theory means that the stress-induced velocity variation should constantly remain zero. Knowing that the air temperature during this period equals $22.0 \pm 0.5^{\circ} \mathrm{C}$, the average values were calculated using the standard error format from CWI results both with and without bias control (i.e. $\alpha_{F}$ and $\alpha_{\text {test }}$ ) for this 8-min period (results shown in Table 2 ). The reduction in both average value and standard error demonstrates that with this bias control technique, CWI analysis is subject to less interference by ambient temperature fluctuations, hence improving the accuracy of results.

The results posted in Fig. 6 further demonstrate the role of our bias control technique in the study of acoustoelastic phenomena. When studying the acoustoelastic effect with a uniaxial tensile load, all three tests were performed with identical loading procedures, during which the tensile force had been increased by $6 \mathrm{kN}$ (with a constant rate of $32 \mathrm{kN} / \mathrm{hr}$ ) (Fig. 6a). Though the air temperature fluctuation in Fig. $6 \mathrm{~b}$ is quite different for every test. The curves in Fig. $6 c$ and d associate the velocity variation evaluated respectively without $\left(\alpha_{\text {test }}\right)$ and with bias control $\left(\alpha_{F}\right)$ during three loading tests.

The $\alpha_{\text {test }}$ in Fig. 6c decreases during every test; this observation agrees with acoustoelastic theory as the tensile force increases. Their paths differ however due to differences in air temperature fluctuation. By referring to Fig. $6 \mathrm{~b}$ and $\mathrm{c}$, the impact of temperature fluctuation on propagation velocity can be readily observed. In comparison with the results of $\alpha_{\text {test }}$, three $\alpha_{F}$ curves in Fig. $6 \mathrm{~d}$ nearly overlap, which offers greater consistency with acoustoelastic theory and shows satisfactory test repeatability.

In accordance with acoustoelastic theory (Eq. (6)), a linear regression was performed in order to calculate the slopes of CWI results both without and with bias control, as respectively $\Delta \alpha_{\text {test }}$ l $\Delta F$ and $\Delta \alpha_{F} / \Delta F$. Two average slopes were then calculated (average value \pm standard error) from CWI results with and without bias control as $\Delta \alpha_{F} \mid \Delta F$ and $\Delta \alpha_{\text {test }} / \Delta F$, their values are detailed in Table 3. Without the bias control technique, the average value of $\Delta \alpha_{\text {test }} / \Delta F$ is slightly higher (5\%) than the $\Delta \alpha_{F} \mid \Delta F$ value. Nonetheless, a standard error of $\pm 15.5 \%$ (in relative terms) is still present, and this is much higher than the standard error with bias control ( $\pm 2.7 \%$ ), thus lowering the level of accuracy and reliability. The $\Delta \alpha / \Delta F$ slope represents the propagation velocity sensitivity to the applied load in concrete; this measure is used in acoustoelasticity to determine the effective acoustoelastic coefficient $\beta$ (Eq. (6)). Since the bias control technique effectively eliminated the thermally-induced

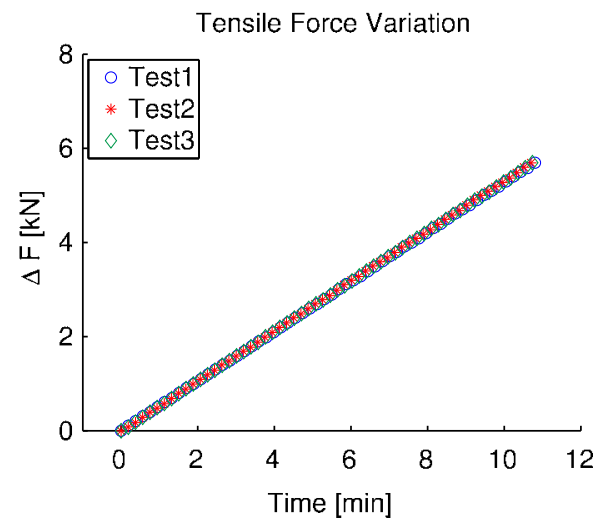

(a)

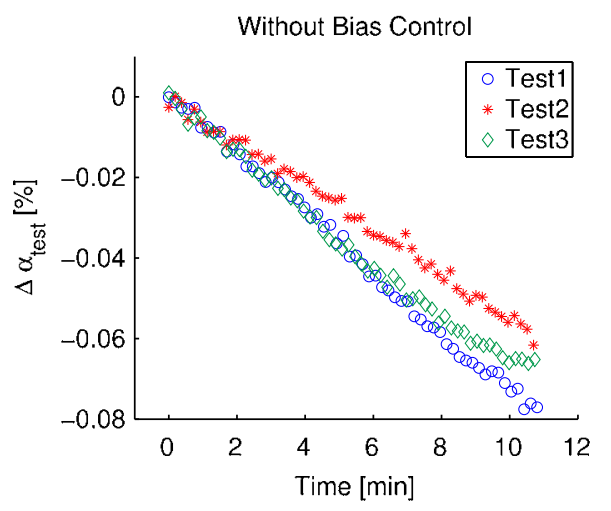

(c)

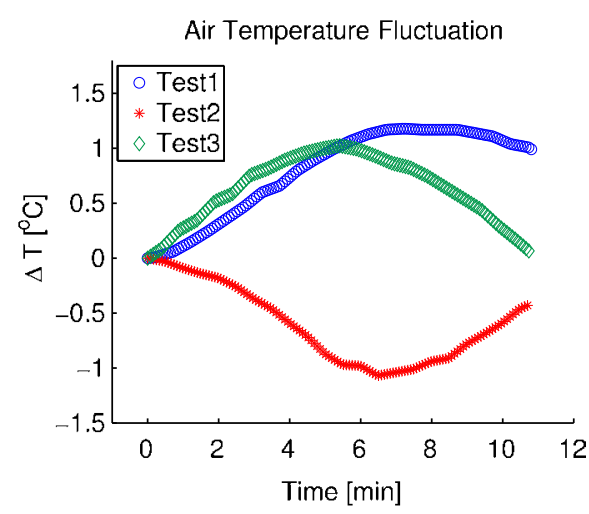

(b)

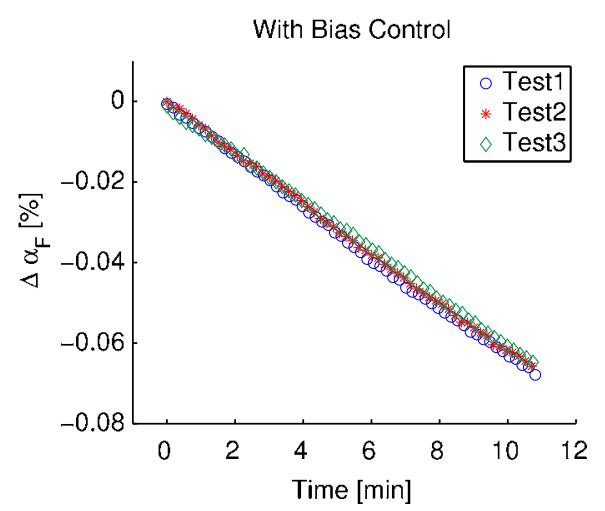

(d)

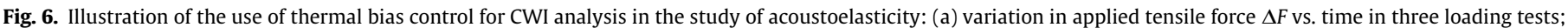

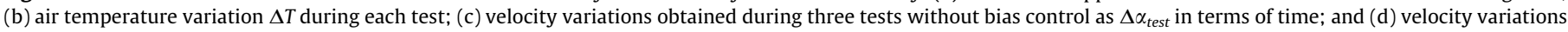
obtained from three tests with bias control as $\Delta \alpha_{F}$ in terms of time. 
Table 3

Slopes of $\Delta \alpha / \Delta F$ and the average values computed from CWI analysis results both with and without bias control, as respectively $\Delta \alpha_{F} \mid \Delta F$ and $\Delta \alpha_{\text {test }} / \Delta F$.

\begin{tabular}{lllll}
\hline$(\% / \mathrm{kN})$ & Average & Test 1 & Test 2 & Test 3 \\
\hline$\Delta \alpha_{F} / \Delta F$ & $-0.01159 \pm 0.00032$ & -0.01190 & -0.01165 & -0.01124 \\
$\Delta \alpha_{\text {test }} / \Delta F$ & $-0.01223 \pm 0.00190$ & -0.01411 & -0.01030 & -0.01227
\end{tabular}

velocity variation (Fig. 6), $\Delta \alpha_{F} / \Delta F$ provides a more reliable estimation of the effective acoustoelastic coefficient $\beta$. As an intrinsic nonlinear parameter, $\beta$ indicates the material's nonlinear elastic properties, which are sensitive to microscopic damage. A reliable and accurate assessment of $\beta$ serves as the foundation of an early-stage damage detection of concrete (see [34] for its application to the study of stress-induced velocity variation and damage detection in concrete).

\section{Conclusion}

This research has presented an alternative bias control technique for CWI analysis. With a specific experimental protocol utilizing a reference specimen, this technique can be easily implemented under laboratory conditions. Experimental results indicate its high efficiency as regards reducing the bias from both experimental procedures and environmental temperature fluctuations. In the illustration provided in Fig. 6, the bias might not necessarily undermine the analysis since the difference in averaged slope $(\Delta \alpha \mid \Delta F)$ only amounts to $5 \%$. However, the reduction in standard error from $\pm 15.5 \%$ to $\pm 2.7 \%$ (in relative terms) shows that CWI results are far more accurate when using our bias control technique. It must also be noted that these experiments were performed with controlled air temperature whose variations were limited to $\pm 2{ }^{\circ} \mathrm{C}$. For the CWI analysis involving measurements with specimens exposed to an uncontrolled ambient temperature, generating potentially large temperature fluctuations [8], this bias control technique was able to provide more reliable and accurate results. This technique obviously is not currently applicable for in situ measurements, since in most instances a reference specimen is lacking. Nevertheless, we think this technique might still be found useful in certain cases where structures present (1) temporal-variation of localized stress and (2) quasi-uniformly distributed spatial gradient of temperature, for example a oil reservoir.

\section{Acknowledgements}

This research has been supported by the ECND-PdL (Loire Valley cluster for Non-Destructive Evaluation and Control), which is financed by the Loire Valley Regional government (France). Our thanks are extended to Robert Sachs, a native English speaker commissioned to proofread the final version of this paper.

\section{References}

[1] G. Poupinet, W.L. Ellsworth, J. Fréchet, Monitoring velocity variations in the crust using earthquake doublets: an application to the Calaveras fault, California, J. Geophys. Res. 89 (1984) 5719-5731.

[2] P.M. Roberts, W.S. Phillips, M.C. Fehler, Development of the active doublet method for measuring small velocity and attenuation changes in solids, J. Acoust. Soc. Am. 91 (1992) 3291-3302.

[3] R. Snieder, A. Grt, H. Douma, J. Scales, Coda wave interferometry for estimating nonlinear behavior in seismic velocity, Science 295 (2002) 2253-2255.

[4] A. Gret, R. Snieder, J. Scales, Time-lapse monitoring of rock properties with coda wave interferometry, J. Geophys. Res. 111 (2006).
[5] A. Gret, R. Snieder, U. Ozbay, Monitoring in situ stress changes in a mining environment with coda wave interferometry, Geophys. J. Int. 167 (2006) 504508.

[6] C. Payan, V. Garnier, J. Moysan, P.A. Johnson, Determination of third order elastic constants in a complex solid applying coda wave interferometry, Appl. Phys. Lett. 94 (2009) 011904.

[7] E. Larose, S. Hall, Monitoring stress related velocity variation in concrete with a $2 \times 10^{-5}$ relative resolution using diffuse ultrasound, J. Acoust. Soc. Am. 125 (2009) 1853-1856

[8] E. Larose, J. de Rosny, L. Margerin, D. Anache, P. Gouedard, M. Campillo, B. van Tiggelen, Observation of multiple scattering of khz vibrations in a concrete structure and application to monitoring weak changes, Phys. Rev. E 73 (2006) 016609.

[9] R. Snieder, Coda wave interferometry and the equilibration of energy in elastic media, Phys. Rev. E 66 (2002) 046615.

[10] R.L. Weaver, O. Lobkis, Temperature dependence of diffuse field phase, J. Acoust. Soc. Am. 106 (1999) 2203.

[11] O.I. Lobkis, R.L. Weaver, Coda-wave interferometry in finite solids: Recovery of p-to-s conversion rates in an elastodynamic billiard, Phys. Rev. Lett. 90 (2003) 254302.

[12] Y. Lu, J.E. Michaels, A methodology for structural health monitoring with diffuse ultrasonic waves in the presence of temperature variations, Ultrasonics 43 (2005) 717-731.

[13] $\mathrm{T}$. $\mathrm{Wu}, \mathrm{T}$. Lin, The stress effect on the ultrasonic velocity variations of concrete under repeated loading, ACI Mater. J. 95 (5) (1998) 519-524.

[14] S. Stahler, E. Niederleithinger, C. Sens-Schönfelder, Monitoring stress changes in a concrete bridge with coda wave interferometry, J. Acoust. Soc. Am. 129 (2011) 1945-1952.

[15] A.A. Shah, S. Hirose, Nonlinear ultrasonic investigation of concrete damaged under uniaxial compression step loading. J. Mater. Civ. Eng. 22 (2010) 476484

[16] P. Antonaci, C. Bruno, A. Gliozzi, M. Scalerandi, Monitoring evolution of compressive damage in concrete with linear and nonlinear ultrasonic methods, Cement Concrete Res. 40 (2010) 1106-1113.

[17] C. Hadziioannou, E. Larose, O. Coutant, P. Roux, M. Campillo, Stability of monitoring weak changes in multiply scattering media with ambient noise correlation: laboratory experiments, J. Acoust. Soc. Am. 125 (2009) 36883695.

[18] R.L. Weaver, On diffuse waves in solid media, J. Acoust. Soc. Am. 71 (1982) $1608-1609$.

[19] R.L. Weaver, Diffuse elastic waves at a free surface, J. Acoust. Soc. Am. 78 (1985) 131-136.

[20] V. Garnier, B. Piwakowski, O. Abraham, G. Villain, C. Payan, J. Chaix, Acoustical techniques for concrete evaluation: Improvements, comparisons and consistencies, Constr. Build. Mater., submitted for publication.

[21] C. Sens-Scheonfelder, E. Larose, Temporal changes in the lunar soil from correlation of diffuse vibrations, Phys. Rev. E 78 (2008) 045601.

[22] J.L. Rodgers, W.A. Nicewander, Thirteen ways to look at the correlation coefficient, Am. Stat. 42 (1988) 59-66.

[23] A. Derode, A. Tourin, M. Fink, Random multiple scattering of ultrasound. I. Coherent and ballistic waves, Phys. Rev. E 64 (2001) 036605.

[24] A. Tourin, A. Derode, A. Peyre, M. Fink, Transport parameters for an ultrasonic pulsed wave propagating in a multiple scattering medium, J. Acoust. Soc. Am. 108 (2000) 503-512.

[25] Y. Hamiel, V. Lyakhovsky, Y. Ben-Zion, The elastic strain energy of damaged solids with applications to non-linear deformation of crystalline rocks, Pure Appl. Geophys. 168 (2011) 2199-2210. 10.1007/s00024-011-0265-7.

[26] F.D. Murnaghan, Finite deformations of an elastic solid, Am. J. Math. 59 (1937) 235.

[27] D.S. Hughes, J.L. Kelly, Second-order elastic deformation of solids, Phys. Rev. 92 (1953) 1145-1149.

[28] D.M. Egle, D.E. Bray, Measurement of acoustoelastic and third-order elastic constants for rail steel, J. Acoust. Soc. Am. 60 (1976) 741-744.

[29] I. Lillamand, J.-F. Chaix, M.-A. Ploix, V. Garnier, Acoustoelastic effect in concrete material under uni-axial compressive loading, NDT E Int. 43 (2010) 655-660.

[30] P. Shokouhi, A. Zoga, H. Wiggenhauser, Nondestructive investigation of stressinduced damage in concrete, Adv. Civ. Eng. 2010 (2010) 9 (Article ID 740189).

[31] Y. Zhang, O. Abraham, E. Larose, T. Planes, A. Le Duff, B. Lascoup, V. Tournat, R. El Guerjouma, L.-M. Cottineau, O. Durand, Following stress level modification of real size concrete structure with coda wave interferometry (CWI), AIP Conf Proc. 1335 (2011) 1291-1298.

[32] A. Mazzeranghi, D. Vangi, Methodology for minimizing effects of temperature in monitoring with the acousto-ultrasonic technique, Exp. Mech. 39 (1999) 86-91. 10.1007/BF0233111.

[33] F. Deroo, J.-Y. Kim, J. Qu, K. Sabra, L.J. Jacobs, Detection of damage in concrete using diffuse ultrasound, J. Acoust. Soc. Am. 127 (2010) 3315-3318.

[34] Y. Zhang, O. Abraham, F. Grondin, A. Loukili, V. Tournat, A. Le Duff, B. Lascoup, O. Durand, Study of Stress-Induced Velocity Variation and Damage Detection in Concrete Under Direct Tensile Force by using Thermally-Compensated Coda Wave Interferometry, Ultrasonics 52 (2012) 1038-1045. 